\title{
Quantitative assessment of SARS-CoV-2 virus in nasopharyngeal swabs stored in transport medium by a straightforward LC-MS/MS assay targeting Nucleocapsid, Membrane and Spike proteins
}

\section{AUTHORS :}

Justyna SAADI ${ }^{a}$, Saoussen OUESLATI ${ }^{b}$, Laurent BELLANGER', Fabrice GALLAISc, Laurent DORTET ${ }^{\mathrm{b}}$ AnneMarie ROQUE-AFONSO ${ }^{d}$, Christophe JUNOT ${ }^{a}$, Thierry NAAS ${ }^{b}$ François FENAILLEa ${ }^{*}$, François BECHER ${ }^{{ }^{*}}$

* These authors share senior co-authorship.

\section{AFFILIATIONS:}

a Université Paris-Saclay, CEA, INRAE, Département Médicaments et Technologies pour la Santé (DMTS), SPI, 91191 Gif sur Yvette, France

${ }^{b}$ Bacteriology-Hygiene unit, Hôpital Bicêtre, APHP Paris Saclay, Team ReSIST, INSERM U1184, Université Paris-Saclay, LabEx LERMIT, Le Kremlin-Bicêtre, France

' Université Paris-Saclay, CEA, INRAE, Département Médicaments et Technologies pour la Santé (DMTS), SPI, 30200 Bagnols-sur-Cèze, France

${ }^{d}$ Service de Virologie, Hôpital Paul-Brousse, APHP Paris Saclay, and UMR 1193 Physiopathogénèse et traitement des maladies du foie, Villejuif

CORRESPONDING AUTHORS:

Telephone number: +33 1690813 15. Electronic address: francois.becher@cea.fr Telephone number: +33 1690879 54. Electronic address: francois.fenaille@cea.fr

KEYWORDS: SARS-CoV-2, targeted mass spectrometry, sensitivity, robustness, nasal swabs, clinical applications 


\section{Table of contents}

Table S1: Bottom-up proteomic of a purified virus solution obtained from Vero E6 cells infected with a SARS-CoV-2 reference strain virus (using MaxQuant v1.6.10.43)

Table S2: Total protein concentration and albumin abundance in 4 representative virus transport

media commonly used in hospitals: eSwab, UTM-RT, MicroTest M4RT and Sigma VIROCULT

Table S3: Recovery of the purified virus in PBS-BSA, eSwab, UTM-RT, MicroTest M4RT and Sigma VIROCULT

Figure S1: Comparison of peptide intensity using sequencing grade modified and unmodified trypsin 
Table S1: Bottom-up proteomic of a purified virus solution obtained from Vero E6 cells infected with a SARS-CoV-2 reference strain virus (using MaxQuant v1.6.10.43)

Peptides selected for PRM detection are highlighted in yellow.

sp |PODTC2 |SPIKE_SARS2 Spike glycoprotein OS=Severe acute respiratory syndrome coronavirus 2 OX=2697049 GN=S PE=1 SV=1

\begin{tabular}{|c|c|c|c|c|c|c|c|c|c|c|c|c|}
\hline \multirow{2}{*}{ Peptide Sequence } & \multirow[b]{2}{*}{$\begin{array}{l}\text { A.A } \\
\text { before }\end{array}$} & \multirow{2}{*}{$\begin{array}{l}\text { A.A } \\
\text { after }\end{array}$} & \multirow{2}{*}{ Length } & \multirow{2}{*}{$\begin{array}{l}\text { Missed } \\
\text { cleavages }\end{array}$} & \multirow{2}{*}{ Mass } & \multirow{2}{*}{ Matching Protein } & \multirow{2}{*}{$\begin{array}{c}\text { Start } \\
\text { position }\end{array}$} & \multirow{2}{*}{$\begin{array}{c}\text { End } \\
\text { position }\end{array}$} & & \multirow{2}{*}{$\begin{array}{c}33.8 \\
\text { Intensity }\end{array}$} \\
\hline & & & & & & & & & $z$ & $\begin{array}{l}\text { Posterior } \\
\text { Error Prob } \\
\text { (PEP) }\end{array}$ & $\begin{array}{l}\text { Peptide } \\
\text { Id Score }\end{array}$ & \\
\hline ALTGIAVEQDK & $\mathrm{R}$ & $\mathrm{N}$ & 11 & 0 & 1143.6136 & sp|PODTC2|SPIKE_SARS2 & 766 & 776 & 2 & 0.0010967 & 30.254 & 1317300 \\
\hline $\begin{array}{l}\text { ALTGIAVEQDKNTQEV } \\
\text { FAQVK }\end{array}$ & $\mathrm{R}$ & $Q$ & 21 & 1 & 2288.2012 & sp|PODTC2|SPIKE_SARS2 & 766 & 786 & 3 & $3.48 \mathrm{E}-07$ & 57.745 & 3078100 \\
\hline CYGVSPTK & K & $\mathrm{L}$ & 8 & 0 & 910.42185 & sp|PODTC2|SPIKE_SARS2 & 379 & 386 & 2 & 0.0054459 & 23.019 & 652130 \\
\hline DIADTTDAVR & $\mathrm{R}$ & $\mathrm{D}$ & 10 & 0 & 1075.5146 & sp|PODTC2|SPIKE_SARS2 & 568 & 577 & 2 & 0.0011731 & 31.074 & 656100 \\
\hline DLICAQK & $\mathrm{R}$ & $\mathrm{F}$ & 7 & 0 & 846.42694 & sp|PODTC2|SPIKE_SARS2 & 848 & 854 & 2 & 0.00328 & 26.109 & 1742700 \\
\hline FASVYAWNR & $\mathrm{R}$ & K & 9 & 0 & 1112.5403 & sp|PODTC2|SPIKE_SARS2 & 347 & 355 & 2 & 0.015093 & 17.386 & 353310 \\
\hline FDEDDSEPVLK & K & G & 11 & 0 & 1292.5772 & sp|PODTC2|SPIKE_SARS2 & 1256 & 1266 & 2 & $7.55 \mathrm{E}-11$ & 69.954 & 1230100 \\
\hline FLPFQQFGR & K & $\mathrm{D}$ & 9 & 0 & 1138.5924 & sp|PODTC2|SPIKE_SARS2 & 559 & 567 & 2 & $2.17 \mathrm{E}-06$ & 48.504 & 2265100 \\
\hline FNGIGVTQNVLYENQK & $\mathrm{R}$ & $\mathrm{L}$ & 16 & 0 & 1822.9214 & sp|PODTC2|SPIKE_SARS2 & 906 & 921 & 3 & 0.019297 & 9.9211 & 504060 \\
\hline FQTLLALHR & $\mathrm{R}$ & $S$ & 9 & 0 & 1097.6346 & sp|PODTC2|SPIKE_SARS2 & 238 & 246 & 3 & $2.62 \mathrm{E}-06$ & 46.158 & 3033500 \\
\hline GIYQTSNFR & K & V & 9 & 0 & 1084.5302 & sp|PODTC2|SPIKE_SARS2 & 311 & 319 & 2 & $4.42 \mathrm{E}-05$ & 42.109 & 1328200 \\
\hline GVYYPDK & $\mathrm{R}$ & v & 7 & 0 & 840.40177 & sp|PODTC2|SPIKE_SARS2 & 35 & 41 & 2 & 0.0020066 & 32.47 & 1061700 \\
\hline GWIFGTTLDSK & $\mathrm{R}$ & $T$ & 11 & 0 & 1223.6186 & sp|PODTC2|SPIKE_SARS2 & 103 & 113 & 2 & 0.0002227 & 40.002 & 2420100 \\
\hline $\begin{array}{l}\text { GYHLMSFPQSAPHGV } \\
\text { VFLHVTYVPAQEK }\end{array}$ & K & $\mathrm{N}$ & 28 & 0 & 3138.5749 & sp|PODTC2|SPIKE_SARS2 & 1046 & 1073 & 5 & 0.02263 & 8.1792 & 1025200 \\
\hline HTPINLVR & K & $\mathrm{D}$ & 8 & 0 & 948.5505 & sp|PODTC2|SPIKE_SARS2 & 207 & 214 & 3 & $3.06 \mathrm{E}-06$ & 45.68 & 1110500 \\
\hline IADYNYK & K & $\mathrm{L}$ & 7 & 0 & 885.42323 & sp|PODTC2|SPIKE_SARS2 & 418 & 424 & 2 & 0.0035022 & 24.998 & 1149800 \\
\hline LIANQFNSAIGK & K & 1 & 12 & 0 & 1274.6983 & sp|PODTC2|SPIKE_SARS2 & 922 & 933 & 2 & 0.0013109 & 28.399 & 640650 \\
\hline LNDLCFTNVYADSFVIR & K & G & 17 & 0 & 2045.9881 & sp|PODTC2|SPIKE_SARS2 & 387 & 403 & $2 ; 3$ & 0.0026042 & 24.041 & 2867300 \\
\hline $\begin{array}{l}\text { LPDDFTGCVIAWNSN } \\
\text { NLDSK }\end{array}$ & K & V & 20 & 0 & 2265.0372 & sp|PODTC2|SPIKE_SARS2 & 425 & 444 & $2 ; 3$ & 0.0013335 & 30.416 & 2152600 \\
\hline LQSLQTYVTQQLIR & $\mathrm{R}$ & A & 14 & 0 & 1689.9414 & sp|PODTC2|SPIKE_SARS2 & 1001 & 1014 & $2 ; 3$ & $2.31 \mathrm{E}-06$ & 45.359 & 3205900 \\
\hline
\end{tabular}




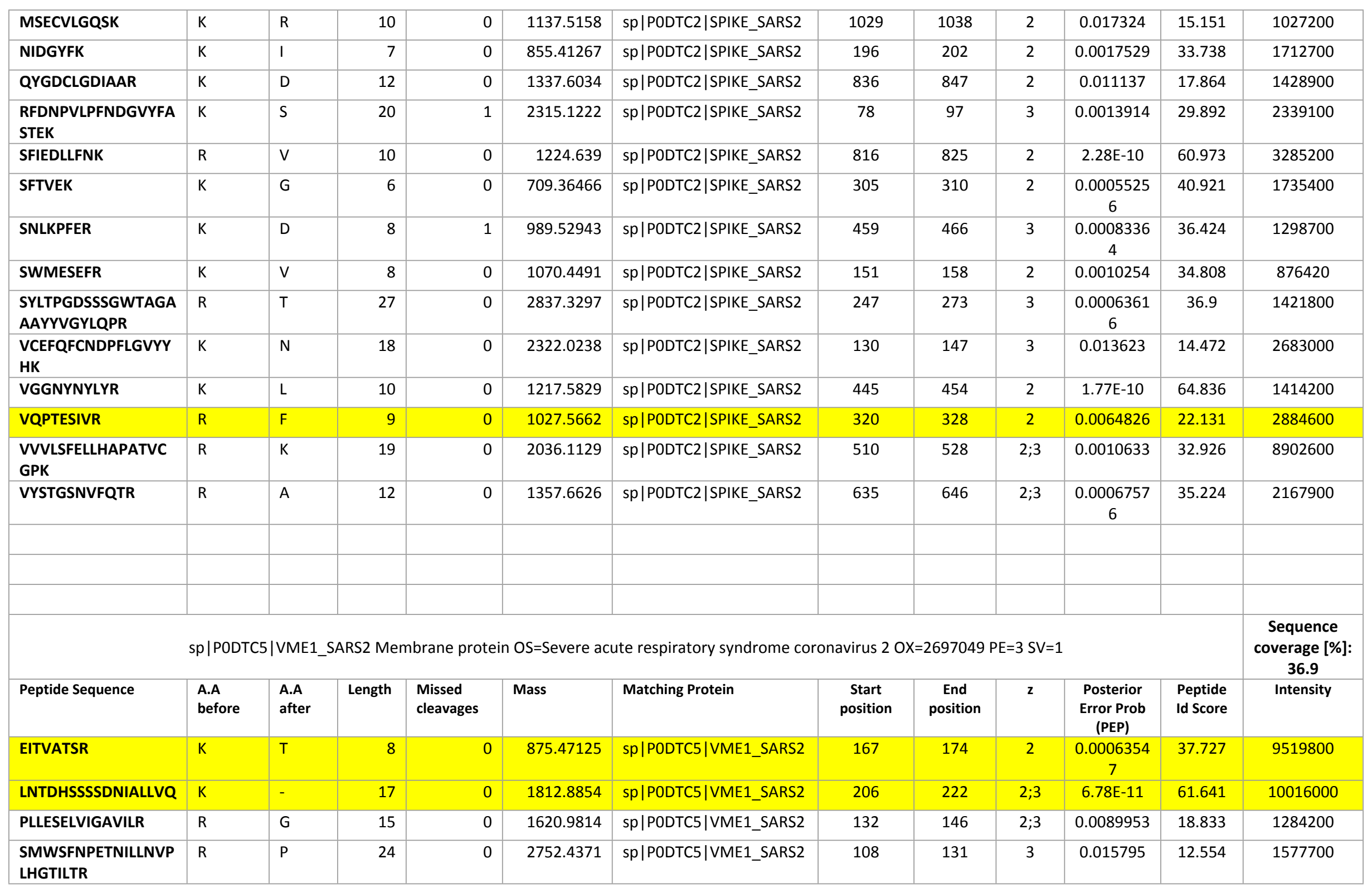




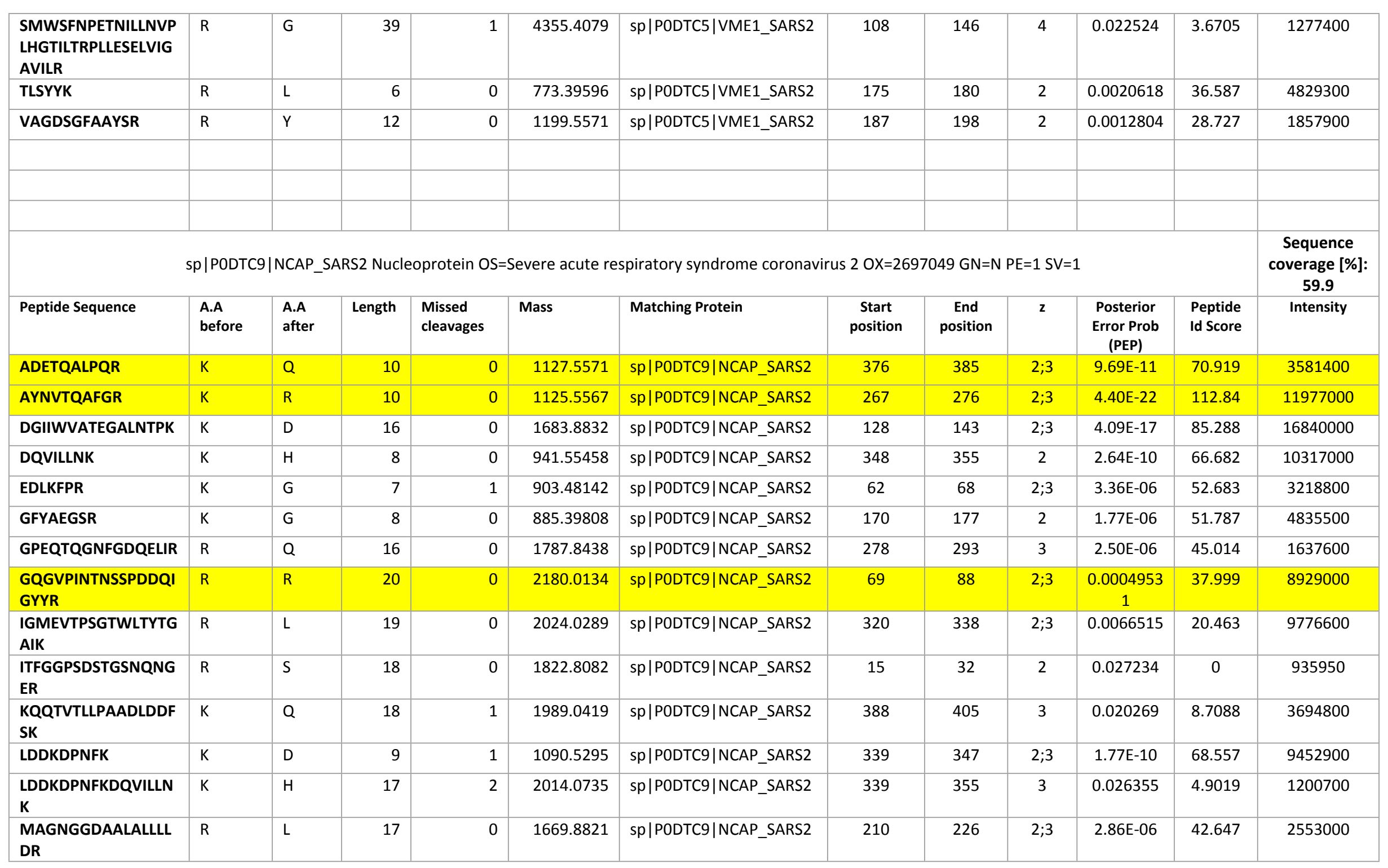




\begin{tabular}{|c|c|c|c|c|c|c|c|c|c|c|c|c|}
\hline $\begin{array}{l}\text { NPANNAAIVLQLPQG } \\
\text { TTLPK }\end{array}$ & $\mathrm{R}$ & G & 20 & 0 & 2059.1426 & sp|PODTC9|NCAP_SARS2 & 150 & 169 & $2 ; 3$ & $6.17 \mathrm{E}-07$ & 55.864 & 19206000 \\
\hline QLQQSMSSADSTQA & K & - & 14 & 0 & 1480.6464 & sp|PODTC9|NCAP_SARS2 & 406 & 419 & 2 & 0.012017 & 16.632 & 1783800 \\
\hline $\begin{array}{l}\text { QQTVTLLPAADLDDFS } \\
\mathrm{K}\end{array}$ & K & Q & 17 & 0 & 1860.9469 & sp|PODTC9|NCAP_SARS2 & 389 & 405 & $2 ; 3$ & 0.0014197 & 29.807 & 8206100 \\
\hline $\begin{array}{l}\text { RPQGLPNNTASWFTA } \\
\text { LTQHGK }\end{array}$ & $R$ & $\mathrm{E}$ & 21 & 1 & 2323.1822 & sp|PODTC9|NCAP_SARS2 & 41 & 61 & $3 ; 4$ & 0.0068123 & 21.2 & 17716000 \\
\hline $\begin{array}{l}\text { WYFYYLGTGPEAGLPY } \\
\text { GANK }\end{array}$ & $\mathrm{R}$ & $\mathrm{D}$ & 20 & 0 & 2266.0735 & sp|PODTC9|NCAP_SARS2 & 108 & 127 & $2 ; 3$ & 0.0002166 & 40.52 & 5574200 \\
\hline
\end{tabular}


Table S2: Total protein concentration and albumin abundance in 4 representative virus transport media commonly used in hospitals: eSwab, UTM-RT, MicroTest M4RT and Sigma VIROCULT

\begin{tabular}{|c|c|c|}
\hline Matrix & $\begin{array}{c}\text { Total } \\
\text { Protein Concentration } \\
(\mu \mathrm{g} / \mathrm{mL})\end{array}$ & $\begin{array}{l}\text { MS signal intensity of } \\
\text { each albumin peptides }\end{array}$ \\
\hline PBS BSA & 1,000 & $>10^{8}$ \\
\hline eSwab & $<500$ & $<10^{5}$ \\
\hline $\begin{array}{c}\text { Sigma } \\
\text { VIROCULT }\end{array}$ & $<500$ & $<10^{5}$ \\
\hline UTM-RT & $\sim 10,000$ & $>10^{8}$ \\
\hline $\begin{array}{l}\text { MicroTest } \\
\text { M4RT }\end{array}$ & $\sim 10,000$ & $>10^{8}$ \\
\hline
\end{tabular}

Total protein concentration was determined by BCA assay after removal of any interfering substances from VTM by methanol precipitation of proteins.

Abundance of bovine albumin was estimated by PRM monitoring of peptides LVNELTEFAK and QTALVELLK 
Table S3: Recovery of the purified virus in PBS-BSA, eSwab, UTM-RT, MicroTest M4RT and Sigma VIROCULT

\begin{tabular}{ccc}
\hline \multirow{2}{*}{ PEPTIDE } & \multicolumn{2}{c}{ NPANNAAIVLQLPQGTTLPK } \\
\cline { 2 - 3 } & MEAN & SD \\
\hline PBS BSA & 77.4 & 13.9 \\
UTM-RT & 85 & 13.5 \\
MicroTest & 87.3 & 31.7 \\
M4RT & 69.7 & 16 \\
eSwab & 55.4 & 16.1 \\
Sigma & & \\
VIROCULT
\end{tabular}

Peak area ratios with labeled peptide in PBS-BSA, eSwab, UTM-RT, MicroTest M4RT and Sigma VIROCULT were compared to the reference signal of the purified virus at the same concentration. 
Figure S1: Comparison of peptide intensity using sequencing grade modified and unmodified trypsin

MS Signal was determined on the swab sample from patient NP29. Signal obtained using unmodified trypsin (left) or sequencing grade trypsin (right) is indicated.

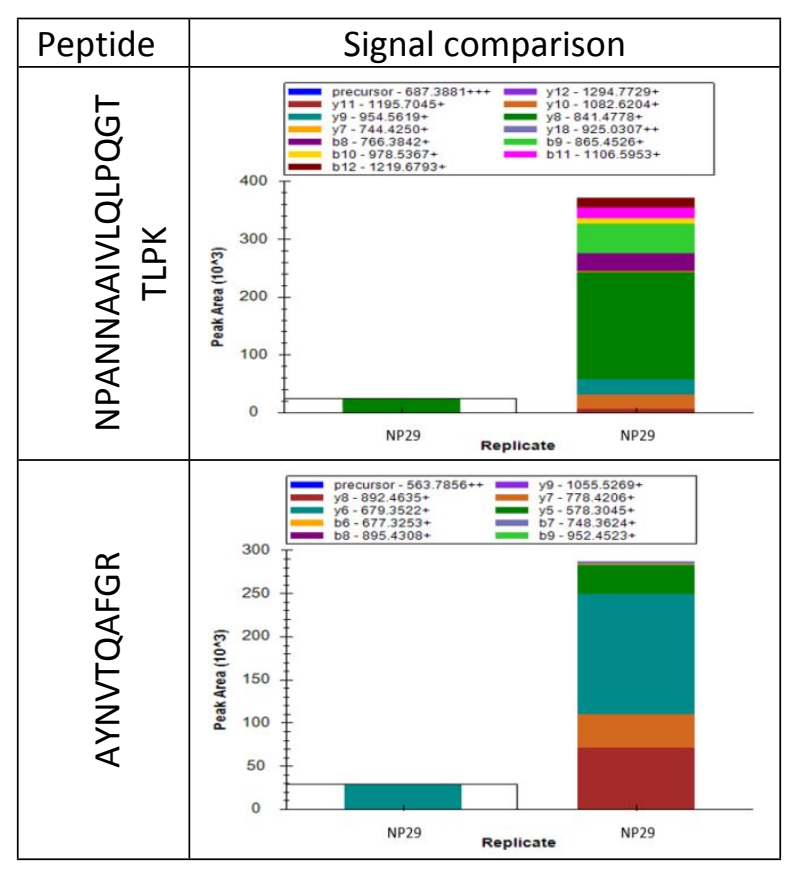

Low or undetectable signal was detected in Skyline for peptides NPANNAAIVLQLPQGTTLPK and AYNVTQAFGR when using unmodified trypsin. 